\title{
A REMARK ON A PAPER BY ABIAN
}

By

M. MORLEY (Ithaca)

A recent paper [1] by A. ABIAN has as its main result that the theory of atomless Boolean algebras is not categorical in certain uncountable powers. This is a weak special case of the following known result (see [3]).

THEOREM. If a theory in the lower predicate calculus has a model with a definable binary relation which linearly orders any infinite subset of that model then it is not categorical in any uncountable power $K$.

In particular, no extension of the theory of Boolean algebras can be categorical in any uncountable power.

For a wide class of cardinals $K$ the above theorem was proved earlier by EHRINFEUCHT [2]. See also footnote 18 of [3].

(Received 3 September 1973)

DEPARTMENT OF MATHEMATICS

WHITE HALL

CORNELL UNTVERSITY

ITHACA, NEW YORK 14850

U.S.A.

\section{References}

[1] A. AbIAn, Nonisomorphic atomless Boolean algebras, Acta Math. Acad. Sci. Hung., 24 (1973), pp. $139-141$.

[2] A. EhrenfeuChT, On theories categorical in power, Fund. Math., 44 (1957), pp. 241-248.

[3] M. Morley, Categoricity in power, Trans. AMS, 114 (1965), pp. 514-538. 